\title{
Potential gains in lifetime net merit from genomic testing of cows, heifers, and calves on commercial dairy farms
}

\author{
K. A. Weigel, ${ }^{* 1}$ P. C. Hoffman, ${ }^{*}$ W. Herring, $†$ and T. J. Lawlor Jr.‡ \\ ${ }^{*}$ Department of Dairy Science, University of Wisconsin, Madison 53706 \\ †Pfizer Animal Health, Kalamazoo, Ml 49001 \\ łHolstein Association USA, Brattleboro, VT 05302
}

\section{ABSTRACT}

The objective of this study was to quantify the gains in genetic potential of replacement females that could be achieved by using genomic testing to facilitate selection and culling decisions on commercial dairy farms. Data were simulated for 100 commercial dairy herds, each with 1,850 cows, heifers, and calves. Parameters of the simulation were based on the US Holstein population, and assumed reliabilities of traditional and genomic predictions matched reliabilities of animals that have been genotyped to date. Selection of the top 10, $20,30, \ldots, 90 \%$ of animals within each age group was based on parent averages and predicted transmitting abilities with or without genomic testing of all animals or subsets of animals that had been presorted by traditional predictions. Average gains in lifetime net merit breeding value of selected females due to genomic testing, minus prorated costs of genotyping the animals and their unselected contemporaries, ranged from $\$ 28$ (top $90 \%$ selected) to $\$ 259$ (top $20 \%$ selected) for heifer calves with no pedigrees, $\$ 14$ (top $90 \%$ selected) to $\$ 121$ (top $10 \%$ selected) for heifer calves with known sires, and $\$ 7$ (top $90 \%$ selected) to $\$ 87$ (top $20 \%$ selected) for heifer calves with full pedigrees. In most cases, gains in genetic merit of selected heifer calves far exceeded prorated genotyping costs, and gains were greater for animals with missing or incomplete pedigree information. Gains in genetic merit due to genomic testing were smaller for lactating cows that had phenotypic records, and in many cases, these gains barely exceeded or failed to exceed genotyping costs. Strategies based on selective genotyping of the top, middle, or bottom $50 \%$ of animals after presorting by traditional parent averages or predicted transmitting abilities were cost effective, particularly when pedigrees or phenotypes were available and a relatively small proportion of animals were to be selected or culled. Based on these re-

Received August 26, 2011.

Accepted November 25, 2011.

${ }^{1}$ Corresponding author: kweigel@wisc.edu sults, it appears that routine genotyping of heifer calves or yearling heifers can be a cost-effective strategy for enhancing the genetic level of replacement females on commercial dairy farms. Increasing the accuracy of predicted breeding values for young females with genomic testing might lead to synergies with other management tools and strategies, such as propagating genetically superior females using advanced reproductive technologies or selling excess females that were generated by the use of sex-enhanced semen.

Key words: genomic testing, replacement heifer, genetic selection

\section{INTRODUCTION}

Since the introduction of the BovineSNP50 (50K) BeadChip by Illumina (San Diego, CA) in late 2007, vast numbers of dairy cattle have been genotyped worldwide, and the resulting genomic information has been fully integrated into national genetic evaluation systems (Hayes et al., 2009; VanRaden et al., 2009). The majority of animals that were genotyped initially were young males and potentially elite females, because the cost of the $50 \mathrm{~K}$ array often precluded its use for routine genomic testing of cows, heifers, and calves on commercial dairy farms. Recent research has shown that genotypes from a low-density array with approximately 3,000 (3K) SNP can be used effectively for imputation of $50 \mathrm{~K}$ genotypes from an ancestral reference population, and genomic-predicted transmitting abilities (GPTA) derived from these imputed genotypes are almost as accurate as $50 \mathrm{~K}$ predictions for a fraction of the cost (Weigel et al., 2010a,b; Zhang and Druet, 2010; Dassonneville et al., 2011). Illumina introduced a 3K bovine SNP array in 2010, and several for-profit companies and non-profit organizations offer genotyping services to dairy farmers based on this platform.

The availability of less expensive genotyping options, such as the Illumina $3 \mathrm{~K}$ array, coupled with the development of a bioinformatics infrastructure that allows efficient and almost transparent integration of the resulting genotypes into the national genetic evalu- 
ation system (Wiggans et al., 2011), is a major step in extending the technology of genomics beyond AI companies and pedigree breeders and into the hands of commercial dairy farmers. However, genotyping a large number of cows, heifers, or calves on a commercial farm represents a significant financial investment. Therefore, cost-benefit studies should assess the financial viability of routine genomic testing and identify genotyping strategies that will maximize gains in the accuracy of selection, culling, mating, and management decisions.

The objective of the present study was to evaluate, via simulation, the expected gains in lifetime net merit (LNM\$) that could be achieved on commercial dairy farms through routine genomic testing of females using a low-density (e.g., 3K) SNP panel, while considering alternative strategies that involve full genotyping or selective genotyping of animals within a given age group.

\section{MATERIALS AND METHODS}

Data were simulated to mimic a commercial dairy operation with 1,000 lactating cows, and 100 replicates were carried out. Each herd contained 1,850 total females that were distributed among 8 age groups: 450 heifer calves ( $<12 \mathrm{mo}), 400$ yearling heifers (12-24 mo), 350 first-parity cows (24-36 mo), 250 second-parity cows (36-48 mo), 170 third-parity cows (48-60 mo), 120 fourth-parity cows (60-72 mo), 70 fifth-parity cows (72-84 mo), and 40 sixth-parity cows (84-96 mo). Parameters of the population were chosen based on the current population of US Holstein cows, as evaluated by the USDA-ARS Animal Improvement Programs Laboratory (Beltsville, MD), with mean PTA for LNM $\$$ of $\$ 45$, standard deviation (SD) of LNM $\$$ equal to $\$ 198$, and genetic trend in LNM $\$$ of $\$ 28$ per year. True transmitting abilities (TA) were generated by sampling from a normal distribution with the aforementioned mean and SD. Values for parent average (PA), PTA, and GPTA included prediction errors that were drawn from a normal distribution with mean zero and SD equal to the product of the square root of assumed reliability (REL) and the genetic SD; assumed REL values are described in the next section. Selection decisions were carried out based on PA, PTA, and GPTA within each age group, so that the potential gains associated with genotyping animals of various ages could be assessed. Whereas TA are typically used to select parents of the next generation, profitability of the current generation is a function of breeding values (BV), which reflect animals' genetic contributions to their own phenotypes. Therefore, TA were converted to BV (i.e., multiplied by 2) before computation of the difference between LNM\$ of animals that were selected based on GPTA and LNM $\$$ of animals from the same age group that were selected based on traditional PA or PTA. The simulation strategy used in this study, which involved simulation of true and estimated breeding values in a single generation, was simplistic compared with alternative approaches, such as simulation of a base population followed by hundreds of generations of random mating or directional selection (e.g., Casellas and Varona, 2011). However, the objective of the present study was not to predict long-term rates of genetic gain or investigate the effects of selection on allele frequencies and other genetic parameters, but rather to generate breeding values for hypothetical dairy herds in which the utility of genomic information could be evaluated within a single generation.

To facilitate an economic assessment of the costs and benefits of genomic testing, the average gain in $\mathrm{BV}$ for LNM\$ was compared with the prorated cost of genotyping per selected individual. The prorated cost of genotyping was computed as the current cost of DNA extraction and 3K SNP genotyping (\$40) times the proportion of animals tested, divided by the product of the percentage of animals retained after testing and the discounted number of times each animal's genetic superiority would be expressed. We assumed that each female would generate her own replacement, and that her daughter and granddaughter would do the same. We further assumed a generation interval of $4 \mathrm{yr}$ and a discount rate of $5 \%$ per year. Therefore, the number of discounted expressions per animal was as follows: $1.00+0.5 \times(1.05)^{-4}+0.25 \times(1.05)^{-8}+0.125 \times$ $(1.05)^{-12}=1.65$. As an example, if all animals within a given age group were genotyped and the top $20 \%$ were selected, the prorated genotyping cost would be $(\$ 40 \times$ $1.0) \times(0.20 \times 1.65)^{-1}=\$ 121.20$. Alternatively, if the bottom $50 \%$ of animals within a given age group were genotyped and the top $90 \%$ were selected, the prorated genotyping cost would be $(\$ 40 \times 0.5) \times(0.90 \times 1.65)^{-1}$ $=\$ 13.47$. Incorporating contributions to future generations into prorated genotyping costs facilitated simple comparisons of genotyping costs with expected gains in LNM $\$$ in the current generation; such comparisons would have been difficult using an alternative approach such as computing cumulative discounted net returns in LNM $\$$ over a 20 -yr planning horizon.

Three classes of animals were considered based on the availability, or lack thereof, of pedigree information: no pedigree, known sire, or full pedigree. Assumed REL values, which are shown in Table 1, were a function of 3 factors: extent of available pedigree information, number of phenotypic records (for lactating cows), and presence or absence of a 3K genotype. Without genomic testing, assumed REL ranged from 0.00 (heifer calves) to 0.27 (sixth-parity cows) for animals with no pedigree information, 0.22 (heifer calves) to 0.48 (sixth-parity cows) 
for animals with known sires, and 0.35 (heifer calves) to 0.58 (sixth-parity cows) for animals with full pedigrees. With genomic testing, assumed REL ranged from 0.48 (heifer calves) to 0.63 (sixth-parity cows) for animals with no pedigree information, 0.58 (heifer calves) to 0.66 (sixth-parity cows) for animals with known sires, and 0.62 (heifer calves) to 0.70 (sixth-parity cows) for animals with full pedigrees. These values were chosen based on mean REL of traditional (pedigree + phenotype) and genomic (pedigree + phenotype + genomic test results) genetic evaluations for US Holstein cows, heifers, and calves that have been genotyped with the Illumina 3K bovine SNP array, as published by the USDA-ARS Animal Improvement Programs Laboratory. Because errors in the recording of parentage information are relatively common on commercial dairy farms, we assumed a rate of $15 \%$ parentage errors in this simulation study. Therefore, $15 \%$ of the animals in each age group were chosen randomly and assigned REL values that reflected an absence of pedigree information (e.g., $0 \%$ for a heifer calf), rather than known sire identification or full pedigree. This reduction in assumed REL increased the prediction error variance and reduced the correlation between $\mathrm{TA}$ and $\mathrm{PA}$ or PTA for these animals.

In practice, the incidence of parentage errors varies widely, and our assumed value of $15 \%$ may not be representative of the quality of pedigree data on some farms. Furthermore, the assumed REL values corresponding to GPTA in Table 1 are based on published values for Holstein cows, heifers, and calves that have been genotyped to date (Wiggans et al., 2011). Many of these were elite animals with genotyped ancestors, and average REL of their GPTA may exceed that of females that would be genotyped on commercial farms, for which few ancestors have been tested. Therefore, a "conservative" simulation was conducted, which assumed a $10 \%$ misidentification rate and reduced the gain in REL due to genomic testing by one-third (e.g.,
0.32 vs. 0.48 for a heifer calf with no pedigree, 0.46 vs. 0.58 for a heifer calf with known sire, and 0.53 vs. 0.62 for a heifer calf with full pedigree, as shown in parentheses in Table 1). Results from this conservative simulation can be used to assess the sensitivity of our analyses and the robustness of our conclusions. After PA, PTA, GPTA, TA, and BV were generated as described above, selection decisions were carried out by ranking animals within each age group, based on their traditional PA (pedigree), their traditional PTA (pedigree + phenotype), or their GPTA (pedigree + phenotype $+3 \mathrm{~K}$ genomic data). In practice, many farmers make selection and culling decisions based on phenotypes for production, health, or conformation. In the present study, we assumed that selection decisions were based on estimated breeding values rather than phenotypes. Whereas this may be a reasonable assumption when sorting calves or yearling heifers, it is likely that actual phenotypes would influence selection and culling decisions involving lactating cows. Nonetheless, we did not attempt to simulate the various ways in which farmers might implement phenotypic selection or culling in the present study.

Several potential strategies are available to commercial dairy producers who seek to improve their herds using genomic testing. Some producers may invest in genotyping all animals of a given age, whereas producers who wish to generate additional income through the sale of breeding stock or accelerate genetic progress by propagating genetically superior females with advanced reproductive technologies could use genomic testing to identify their best animals. On the other hand, herds that have generated extra females by achieving low culling rates or using sex-enhanced semen may wish to reduce feed costs by using genomic testing to identify genetically inferior animals that could be sold early in life or used as embryo recipients. Last, some herds may forego testing of animals that should be culled for reasons such as illness or unacceptable conformation and

Table 1. Reliability values used in the simulation, according to age, pedigree completeness, and use of genomic testing ${ }^{1}$

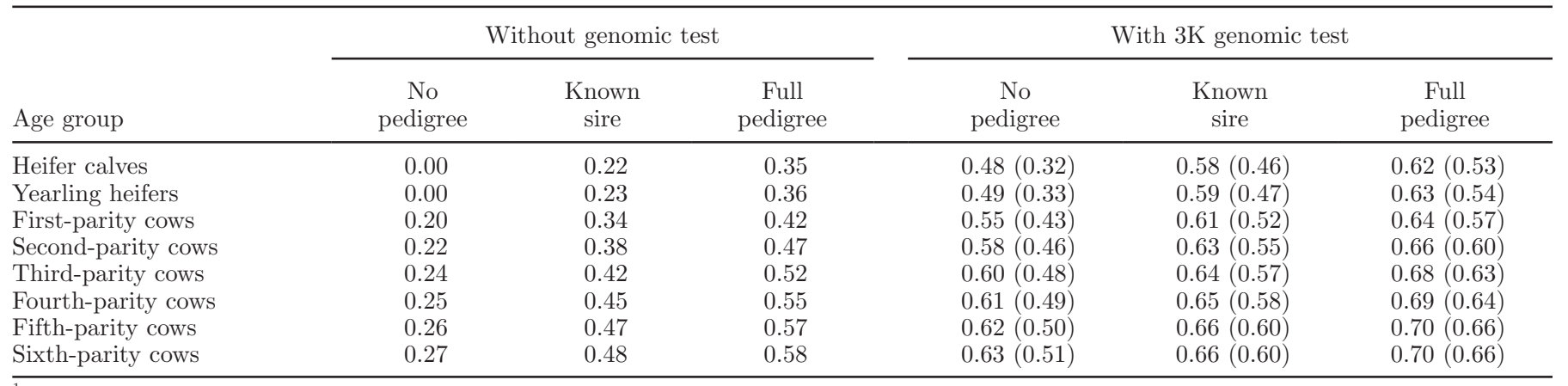

${ }^{1}$ Numbers in parentheses reflect conservative reliability assumptions used in the sensitivity analysis. 
focus on an intermediate group of "borderline" animals, with respect to selection or culling.

In the present study the top $10,20,30, \ldots, 90 \%$ of animals were retained within each age group, and selection decisions were made with or without genomic testing in an effort to illustrate the dynamic relationships between genomic testing and selection decisions. Note that selection and culling are opposite actions; for example, selecting the top $70 \%$ is equivalent to culling the bottom $30 \%$. Some of the selection and culling strategies considered in this simulation study would not be practical on commercial dairy farms. For example, most farmers would run out of replacements if they sold $70 \%$ of their heifer calves, and few farmers would consider culling $50 \%$ of their fourth-lactation cows based on results of a DNA test. However, many farms have generated surplus females by using sex-enhanced semen or reducing involuntary culling, and selling 10, 20 , or $30 \%$ of their calves or yearlings may be feasible. Furthermore, advanced reproductive technologies such as embryo transfer (ET) or in vitro embryo production (IVEP) allow inferior animals to be effectively removed as parents of the next generation, even though these animals may not be physically "culled" from the herd until a suitable replacement becomes available.

Separate analyses were carried out for simulated herds with no pedigrees, known sires, and full pedigrees. Because the cost of genomic testing every animal within a given age group may be prohibitive for some farms, 3 alternative strategies were considered: (1) genotyping the top $50 \%$ of animals after sorting by traditional PA or PTA; (2) genotyping the middle $50 \%$ of animals after sorting by traditional PA or PTA; or (3) genotyping the bottom $50 \%$ of animals after sorting by traditional PA or PTA.

\section{RESULTS AND DISCUSSION}

To date, most studies regarding genomic selection have focused on optimizing strategies for selection of young males and identification of elite females for the purpose of increasing genetic progress within a population for existing or novel traits (e.g., Hansen Axelsson et al., 2011; McHugh et al., 2011; Wensch-Dorendorf et al., 2011). Selection or culling of females on commercial dairy farms has been largely ignored, due to low selection intensity, small selection differentials, and minimal contributions to genetic progress in the "dams of cows" selection pathway (Van Tassell and Van Vleck, 1991). However, the widespread availability of sex-enhanced semen has led to the production of excess females, and enhanced animal well-being on farms that have implemented management improvements such as sand freestalls has led to reduced culling rates. Improvements in management, in conjunction with increases in feed costs, have created an opportunity to revisit the possibility of increasing the overall profitability of commercial farms through genetic selection among lactating cows and replacement heifers. Inexpensive low-density SNP arrays, coupled with subsequent imputation of high-density genotypes and almost seamless integration of genomic data into existing national genetic evaluation systems, can facilitate timely selection among replacement dairy heifers at a level of accuracy that was previously unattainable.

Table 2 shows the average gain in BV for $\mathrm{LNM} \$$ within each age group due to genomic testing, as a function of the percentage of animals retained, after subtracting the prorated cost of testing each selected animal and its unselected contemporaries. The average gain in $\mathrm{BV}$ reflects the difference between the average BV of animals that were selected based on their GPTA and the average BV of animals that were selected based on their PA (calves and yearlings) or PTA (first- through sixth-parity cows). The prorated cost of genotyping, which includes discounted costs attributed to future generations, varies according to the percentage of animals retained. For example, if $20 \%$ of animals are retained, the genetic superiority of each selected animal must offset its own testing fee plus the testing fees of 4 unselected peers. The genotyping strategy that maximized genetic gain of the selected group relative to prorated genotyping costs (i.e., full genotyping or selective genotyping of the top, middle, or bottom $50 \%$ after sorting by traditional PA or PTA) is also shown in Table 2. Keep in mind that selection and culling are opposite actions, so selecting the top $80 \%$ is equivalent to culling the bottom $20 \%$. Furthermore, note that designating an animal for culling does not necessarily mean that the animal is immediately sold for slaughter; the animal could be sold for dairy purposes (immediately or later in life), or it could be used as an embryo recipient. For heifer calves and yearling heifers that lacked pedigrees, genotyping all animals was consistently the best strategy, and gains in genetic merit relative to prorated genotyping costs ranged from $\$ 66$ (top $80 \%$ of calves selected) to $\$ 272$ (top 20\% of yearlings selected). For heifer calves and yearling heifers with known sires, gains ranged from $\$ 27$ (bottom $20 \%$ of yearlings sold) to $\$ 113$ (top $20 \%$ of calves or yearlings selected), and for heifer calves and yearling heifers with full pedigrees, gains ranged from $\$ 19$ (bottom $20 \%$ of calves removed) to $\$ 87$ (top $20 \%$ of calves selected). With known sires or full pedigrees, the best strategy was to genotype the top $50 \%$ of animals when selecting the top $20 \%$. When selecting the top $40 \%$ or top $60 \%$, genotyping all animals was the best strategy, and genotyping the bottom $50 \%$ of 
animals was the best strategy when selecting the top $80 \%$. Adding pedigree information reduces genetic gains relative to genotyping costs, but it also increases our ability to presort animals effectively based on traditional PA before genotyping. Therefore, strategies based on selective genotyping are competitive with full genotyping when pedigree data are available.

Genetic gains and optimal genotyping strategies for first- and second-parity cows that have phenotypes but lack pedigrees were very similar to those of heifer calves and yearling heifers with full pedigrees, as shown in Table 2. As the number of phenotypic records increased, genetic gains relative to genotyping costs decreased. However, this trend was more pronounced among cows with known sires or full pedigrees, because gains in REL due to additional phenotypes were small in the absence of information from relatives. For example, differences between average genetic gains and genotyping costs were $\$ 89$ and $\$ 78$, respectively, for first- and fifth-parity cows with no pedigrees, and $\$ 42$ and $\$ 12$, respectively, for first- and fifth-parity cows with full pedigrees. For lactating cows with known sires or complete pedigrees, selective genotyping of the top, middle, or bottom $50 \%$ consistently provided greater genetic gains relative to prorated genotyping costs than genotyping all cows of a given age. As expected, the difference between genetic gains and prorated genotyping costs was small for cows with multiple phenotypic records and complete pedigrees; for example, the range for fifth-parity cows was from $\$ 3$ (bottom $20 \%$ sold) to $\$ 12$ (top $20 \%$ selected). These data suggest that genomic testing becomes more valuable when less is known about an animal's genetic potential, regardless of whether this knowledge comes from ancestry information or performance data.

Because genomic testing is of greater value when pedigree or performance data are lacking, genomic testing of calves and yearlings is likely to provide greater economic returns than genomic testing of lactating cows. In Figure 1a, the average gain in BV for LNM $\$$ due to genomic testing of heifer calves with unknown ancestry is highlighted. Results are shown as a function of the percentage of heifers retained, utilizing full genotyping or selective genotyping of the top, middle, or bottom $50 \%$ based on traditional PA. The prorated cost of genotyping all heifers or $50 \%$ of the heifers is also shown graphically in Figure 1a. Panels b and c of Figure 1 show detailed results corresponding to 2 potential applications of genomic selection. In Figure $1 \mathrm{~b}$, it is assumed that genomic testing will be used to identify genetically superior heifers that can later be propagated using advanced reproductive technologies, whereas in Figure 1c it is assumed that genomic testing will be used to identify genetically inferior calves that will be sold before rearing costs are incurred.

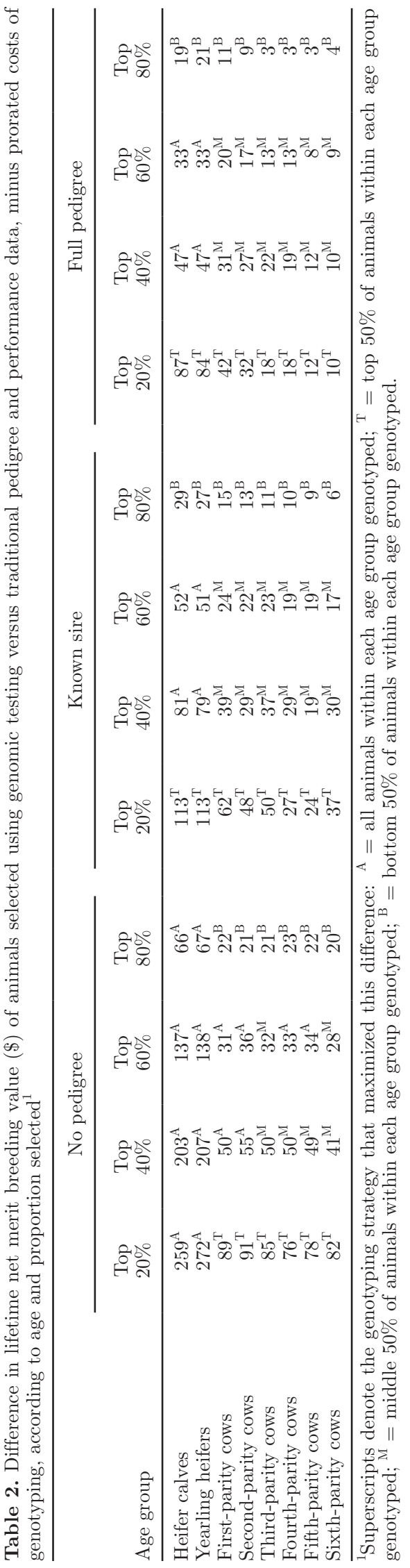

Journal of Dairy Science Vol. 95 No. 4, 2012 
a)

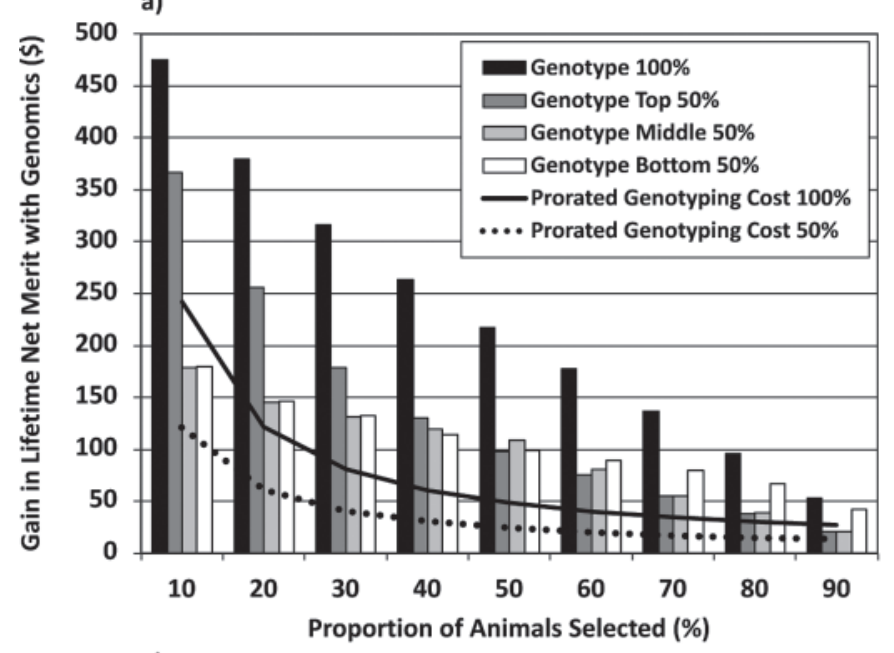

c)

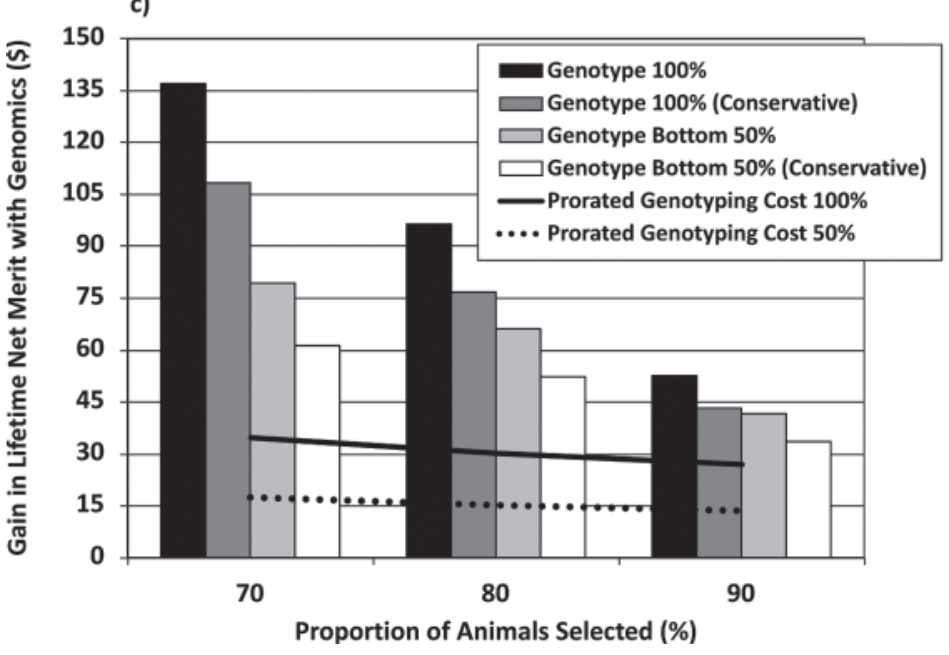

b)

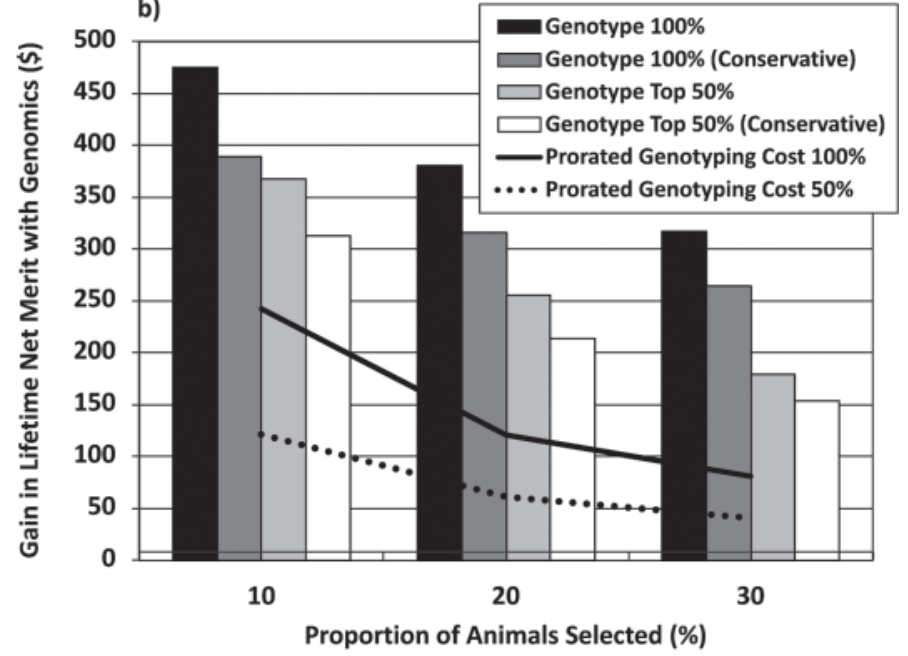

Figure 1. (a) Change in average lifetime net merit breeding value of selected animals due to genomic testing of heifer calves without pedigrees, according to proportion selected and proportion tested. Prorated costs of full $(100 \%)$ and selective (50\%) genotyping are also shown. (b) Detailed view of the change in average lifetime net merit breeding value of selected animals due to genomic testing of heifer calves without pedigrees, with focus on identification of superior breeding stock, according to proportion selected and proportion tested. Prorated costs of full $(100 \%)$ and selective (50\%) genotyping and results of the sensitivity analysis (conservative) are also shown. (c) Detailed view of the change in average lifetime net merit breeding value of selected animals due to genomic testing of heifer calves without pedigrees, with focus on identification of inferior herd replacements, according to proportion selected and proportion tested. Prorated costs of full (100\%) and selective (50\%) genotyping and results of the sensitivity analysis (conservative) are also shown.

Figure 1a shows the expected gains in LNM\$ associated with genomic testing of heifer calves with no pedigrees. This represents an ideal situation, with respect to the benefits of genomic testing, because ancestry data are lacking and phenotypes for important traits will not be available for 2 yr. Genotyping every heifer was cost effective regardless of the proportion selected, because gains associated with genomic testing far exceeded prorated genotyping costs. When all heifers were tested, the average gain in BV was $\$ 316$, $\$ 380$, and $\$ 475$ when the top 30,20 , or $10 \%$ of heifers were selected, respectively, compared with $\$ 137, \$ 96$, and $\$ 53$ when the bottom 30,20,or $10 \%$ of heifers were eliminated, respectively. The prorated cost of genotyping ranged from $\$ 242$ per heifer when the best $10 \%$ of heifers were selected, to $\$ 27$ per heifer when the worst $10 \%$ of heifers were sold.

Figure 1a also shows the results for alternative strategies based on genotyping the top, middle, or bottom $50 \%$ of heifer calves after sorting by their traditional PA. In this case, the traditional PA had 0\% REL (i.e., it was a random number that reflected prediction error). However, when the top 10 to $40 \%$ of heifers were selected, presorting by PA and selective genotyping of the top $50 \%$ generated greater gains in BV than selective genotyping of the middle or bottom $50 \%$. This occurred 
because the subset of heifers with highest PA included (1) heifers with high BV that also had high PA due to random chance; these were confirmed as high genetic merit via genomic testing and retained, and (2) heifers with low BV for which the PA was an overestimate; these were identified as low genetic merit via genomic testing and eliminated. Thus, all heifers that were retained were selected based on genomic testing. By comparison, when the middle or bottom $50 \%$ of heifers were genotyped, some high-merit heifers were identified via genomic testing and added to a selected group that also included low-merit heifers for which the PA was an overestimate (these heifers were selected based on PA without confirmation by genomic testing). The reverse was also true, such that presorting heifers by PA and genotyping the bottom $50 \%$ led to greater gains than genotyping the top or middle $50 \%$ if the bottom 10 to $40 \%$ were removed. In practice, calves and yearlings with neither pedigrees nor phenotypes would not have a PA, and presorting would be impossible. However, many cases occur in which genetically inferior cows or heifers that have limited information rank highly based on errors or biases in their PA or PTA (e.g., due to parentage errors or preferential treatment), and selection of these animals without verifying their genetic merit using genomic testing is not recommended.

Figure $1 \mathrm{~b}$ shows detailed results corresponding to herds that may wish to market breeding stock or implement advanced reproductive technologies to accelerate genetic progress. As noted earlier, selection of the top 30,20 , or $10 \%$ using full genotyping provided average gains of $\$ 316, \$ 380$, and $\$ 475$, respectively, which far exceeded prorated genotyping costs of $\$ 81, \$ 121$, and $\$ 242$, respectively. Even under the conservative assumptions used in our sensitivity analysis, the expected genetic gains were $\$ 263, \$ 316$, and $\$ 389$, respectively. Therefore, it seems that full genotyping of heifers that lack ancestry data to identify high-genetic-merit individuals is warranted, provided that a mechanism exists for marketing or propagating these heifers. Conversely, Figure 1c shows detailed results corresponding to herds that have excess females and wish to reduce feed costs by selling inferior calves before rearing. Again, full genotyping seems to be justified if pedigrees are lacking, because selling the bottom 30,20 , or $10 \%$ of heifers provided genetic gains of $\$ 173, \$ 96$, and $\$ 52$, respectively, compared with prorated genotyping costs of $\$ 35, \$ 30$, and $\$ 27$, respectively. Even with conservative assumptions about the gains in REL that would be provided by genomic testing, the corresponding gains in average net merit were $\$ 108, \$ 77$, and $\$ 43$, respectively, which easily exceeded prorated genotyping costs.

Figure 2a shows the expected gains in LNM $\$$ associated with genomic testing of heifer calves with known sires. In this case, the benefits of genomic testing were smaller, because sire PTA were available for the purpose of ranking selection candidates in the absence of genomic testing. Genotyping all heifers was cost effective if the percentage that was retained ranged from 20 to $80 \%$. However, if the top $10 \%$ of heifers were selected or if the bottom $10 \%$ were removed, the corresponding increase in LNM $\$$ of selected heifers was barely enough to offset genotyping costs. Best results were obtained when 30 to $70 \%$ of heifers were retained. For example, when the top $50 \%$ of heifers were retained, the average gain in BV of selected heifers was $\$ 116$, which far exceeded the prorated genotyping cost of $\$ 48$. When sires were known, presorting of heifers by sire PTA was often more cost effective than full genotyping, particularly when a relatively small proportion (e.g., $\leq 20 \%$ ) were selected or culled. Therefore, it appears that targeting a subset of heifers for genotyping, after presorting by sire PTA, may be a reasonable strategy for herds that do not want to invest in genotyping all heifers or herds that lack subsequent management actions for heifers with intermediate GPTA values.

Figure $2 \mathrm{~b}$ shows detailed results associated with selecting the top 30,20 , or $10 \%$ of heifer calves with known sires; these results correspond to potential applications of genomic testing in herds that wish to market breeding stock or generate extra offspring from superior females. Expected gains in lifetime net merit due to genomic testing were $\$ 169, \$ 206$, and $\$ 254$ when the top 30,20 , or $10 \%$ were selected, respectively, compared with prorated genotyping costs of $\$ 81, \$ 121$, and $\$ 242$. In this case, presorting heifers by traditional PA and genotyping the top $50 \%$ provided corresponding gains of $\$ 121, \$ 174$, and $\$ 242$, respectively, compared with prorated genotyping costs of $\$ 40, \$ 61$, and $\$ 121$. Thus, selective genotyping was more cost effective if only $10 \%$ or $20 \%$ of heifers were retained.

Figure 2c shows results that would be applicable for herds that wish to sell excess heifer calves before rearing. Gains associated with genotyping all heifers and subsequently removing the bottom 30,20 , or $10 \%$ were $\$ 73, \$ 51$, and $\$ 29$, respectively, relative to genotyping costs of $\$ 35, \$ 30$, and $\$ 27$. By comparison, gains from genotyping the bottom $50 \%$ of heifers based on traditional PA and subsequently selling the bottom 30, 20, or $10 \%$ were $\$ 52, \$ 44$, and $\$ 27$, respectively, relative to genotyping costs of $\$ 17, \$ 15$, and $\$ 13$, so once again selective genotyping may be a more cost-effective strategy than full genotyping.

Figure $3 \mathrm{a}$ shows the expected gains in LNM $\$$ that could be realized by genomic testing of heifer calves with full pedigrees. Because more information is known about the genetic merit of each heifer before testing, the gains achieved by full genotyping (if any) were 
a)

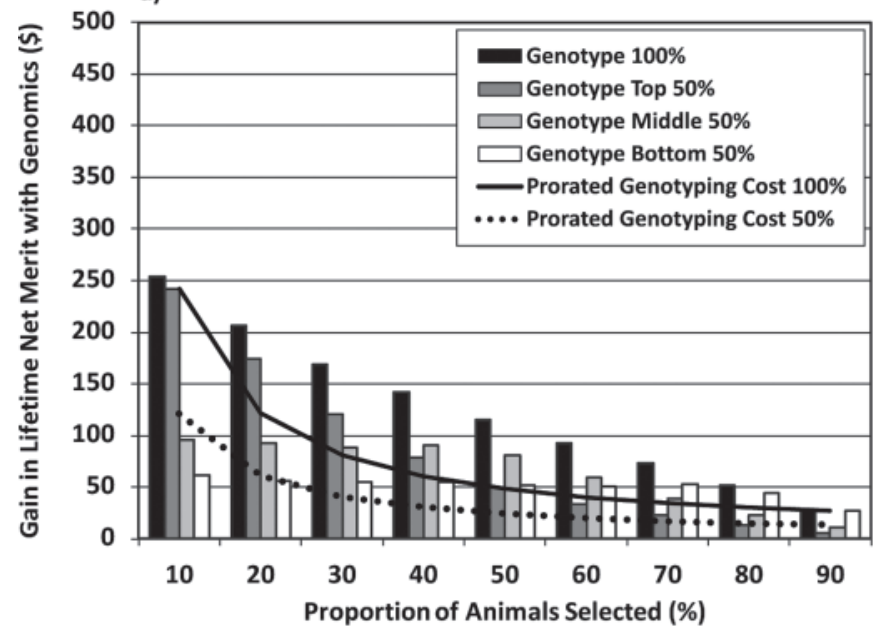

c)

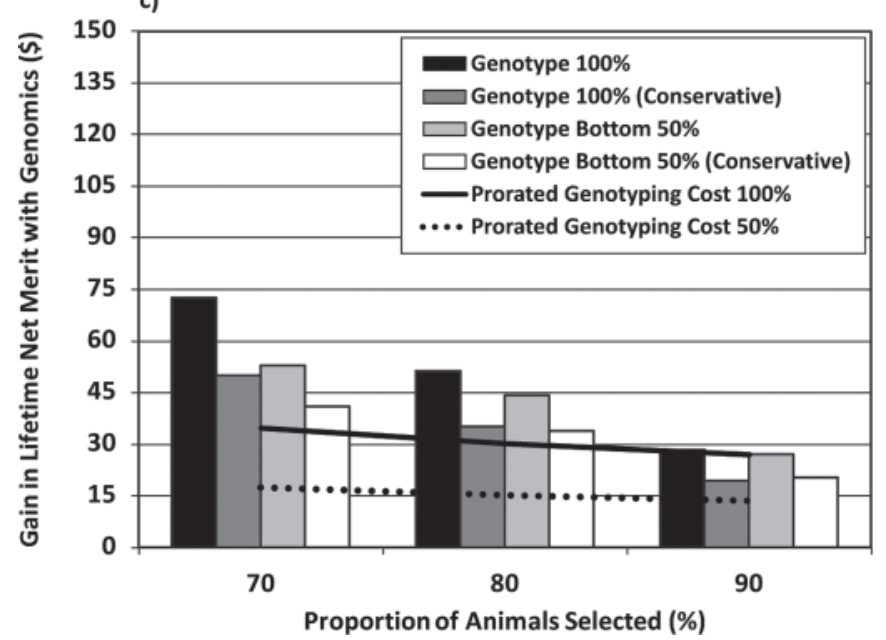

b)

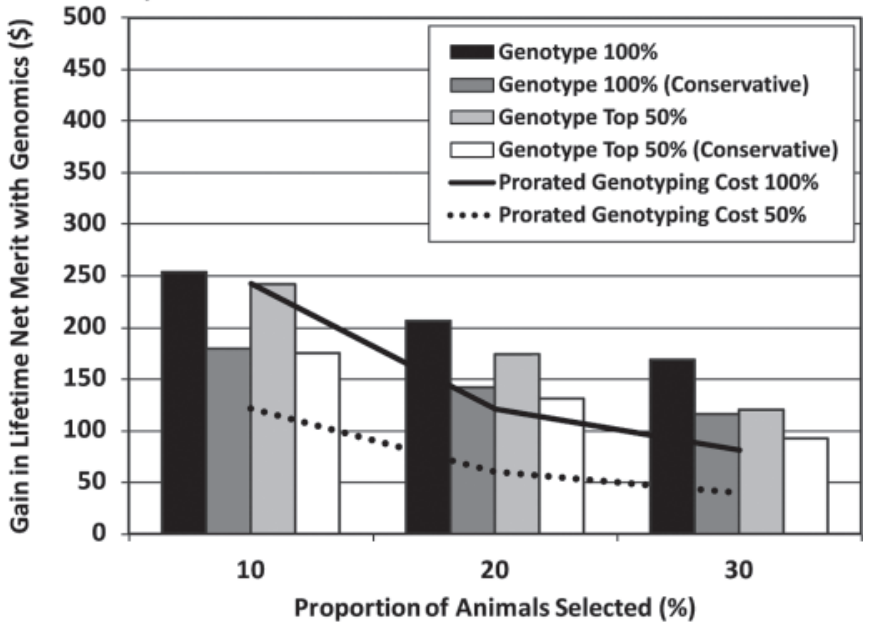

Figure 2. (a) Change in average lifetime net merit breeding value of selected animals due to genomic testing of heifer calves with known sires, according to proportion selected and proportion tested. Prorated costs of full (100\%) and selective (50\%) genotyping are also shown. (b) Detailed view of the change in average lifetime net merit breeding value of selected animals due to genomic testing of heifer calves with known sires, with focus on identification of superior breeding stock, according to proportion selected and proportion tested. Prorated costs of full (100\%) and selective (50\%) genotyping and results of the sensitivity analysis (conservative) are also shown. (c) Detailed view of the change in average lifetime net merit breeding value of selected animals due to genomic testing of heifer calves with known sires, with focus on identification of inferior herd replacements, according to proportion selected and proportion tested. Prorated costs of full (100\%) and selective (50\%) genotyping and results of the sensitivity analysis (conservative) are also shown.

smaller than when pedigree information was lacking or incomplete. In this scenario, genotyping all heifers was cost effective if the goal was to retain 30 to $70 \%$ of the animals. If only $10 \%$ were selected or culled, the gains in LNM\$ were not large enough to offset genotyping costs, and if $20 \%$ were selected or culled, genomic testing was essentially a break-even strategy. Gains were greatest when a moderate proportion of heifers were retained; for example, if the top $50 \%$ of heifers were selected, the average gain in BV of $\$ 90$ per heifer exceeded the prorated genotyping cost of $\$ 48$.

Figure 3b shows detailed results associated with selecting the top 30,20 , or $10 \%$ of heifer calves with full pedigrees. Gains due to genomic testing, which were $\$ 131, \$ 163$, and $\$ 205$, respectively, for full genotyping, and $\$ 104, \$ 148$, and $\$ 201$, respectively, for selective genotyping of the top $50 \%$, were smaller than in the previous scenarios, because more information was known about these heifers before genomic testing. However, presorting of heifers can be done more effectively with complete pedigrees, and the average gains in genetic merit exceeded genotyping costs by $\$ 25$ to $\$ 50$ per heifer when selective genotyping was utilized.

Detailed results associated with removing the bottom 30,20 , or $10 \%$ of heifer calves with full pedigrees are shown in Figure 3c, where average genetic gains were 
a)

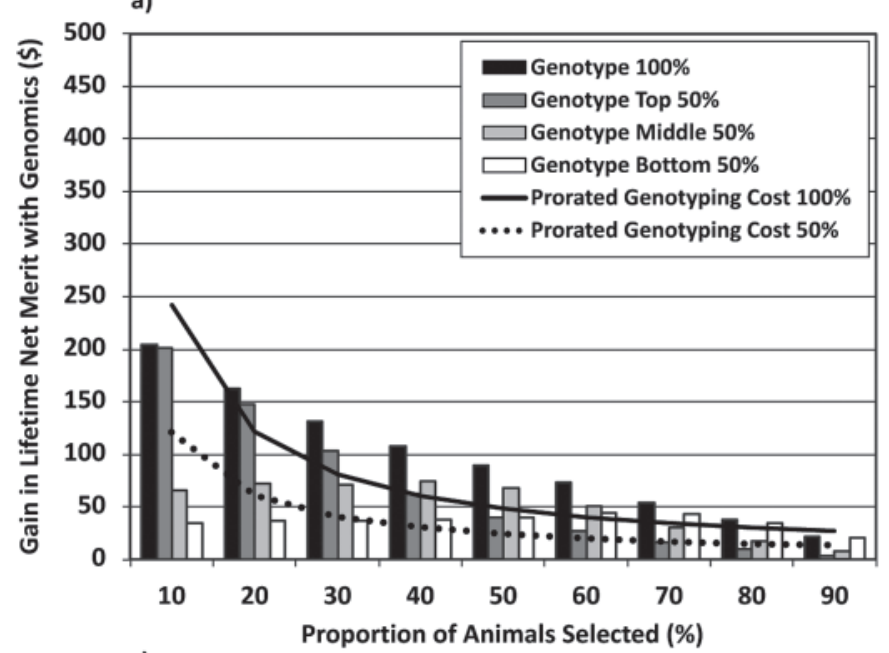

b)

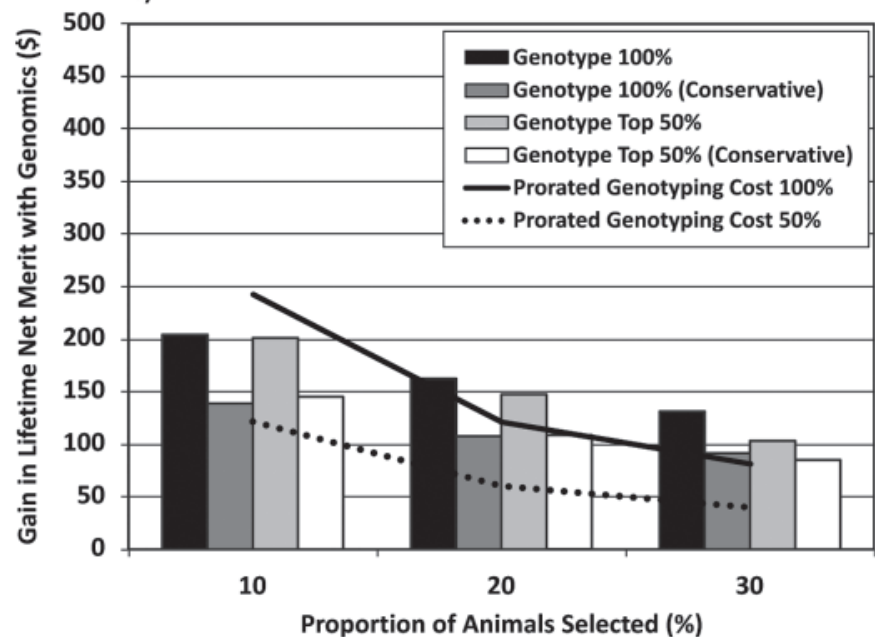

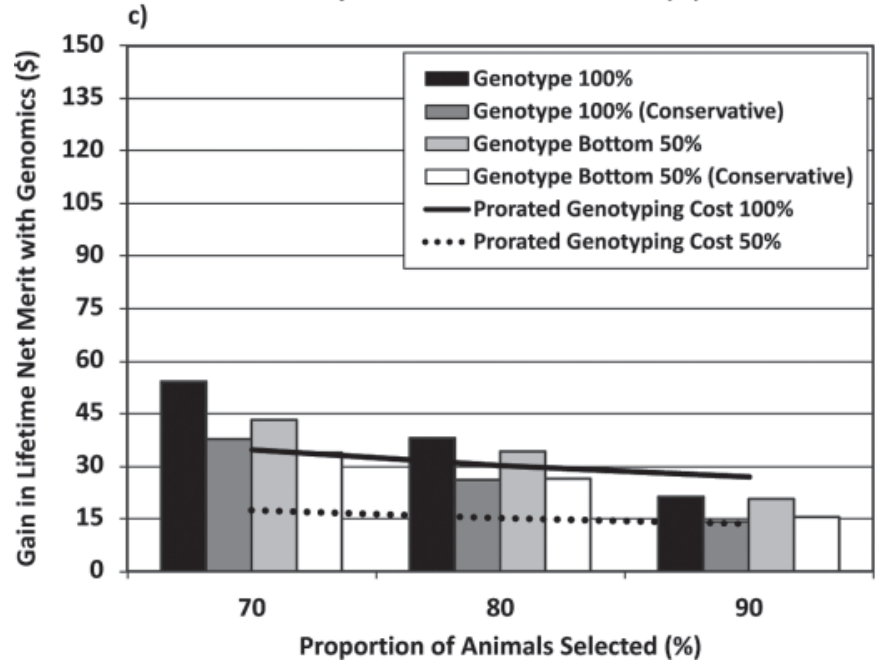

Figure 3. (a) Change in average lifetime net merit breeding value of selected animals due to genomic testing of heifer calves with full pedigrees, according to proportion selected and proportion tested. Prorated costs of full (100\%) and selective (50\%) genotyping are also shown. (b) Detailed view of the change in average lifetime net merit breeding value of selected animals due to genomic testing of heifer calves with full pedigrees, with focus on identification of superior breeding stock, according to proportion selected and proportion tested. Prorated costs of full $(100 \%)$ or selective $(50 \%)$ genotyping and results of the sensitivity analysis (conservative) are also shown. (c) Detailed view of the change in average lifetime net merit breeding value of potentially inferior herd replacements due to genomic testing of heifer calves with full pedigrees, with focus on identification of inferior herd replacements, according to proportion selected and proportion tested. Prorated costs of full (100\%) or selective (50\%) genotyping and results of the sensitivity analysis (conservative) are also shown.

$\$ 21, \$ 38$, and $\$ 54$, respectively, for full genotyping and $\$ 15, \$ 26$, and $\$ 34$, respectively, for selective genotyping of the bottom $50 \%$ based on traditional PA. Genetic gains relative to genotyping costs ranged from $\$ 2$ to $\$ 17$ per heifer when selective genotyping was utilized, and this strategy was typically preferred over full genotyping. It should be noted that small gains in genetic merit over genotyping costs, such as $\$ 10$ to $\$ 15$ per heifer, could be significant when multiplied across a large proportion (e.g., 80 or 90\%) of replacement heifers on a commercial dairy farm. Likewise, genomic information for these heifers could be used for mating decisions (e.g., to minimize inbreeding or avoid inherited genetic defects) or selection of heifers to inseminate with sexenhanced semen; the additional economic benefits associated with these potential applications of genomic information were not considered in this simulation study.

Comparison of Figures 1, 2, and 3 illustrates the importance of maintaining accurate and complete pedigree information for replacement heifers on commercial dairy farms. Figures $1 \mathrm{~b}, 2 \mathrm{~b}$, and $3 \mathrm{~b}$ show comparative results, including results of the sensitivity analysis with conservative REL assumptions, for selecting the top 30, 
20 , or $10 \%$ of heifer calves with no pedigrees, known sires, or full pedigrees, respectively. These results would most appropriately apply to commercial dairy producers who seek to identify their genetically superior heifers at an early age for (1) generating additional income by selling breeding stock, including embryos and AI bull contracts, or (2) increasing the genetic potential of their replacement heifers by using ET or IVEP to rapidly propagate the best females. Figures 1c, 2c, and $3 \mathrm{c}$ show comparative results, including results of the sensitivity analysis, for selling the bottom 30,20 , or $10 \%$ of heifer calves with no pedigrees, known sires, or full pedigrees, respectively. These results would most appropriately apply to commercial dairy producers who seek to cull genetically inferior heifers at a young age to reduce feed costs associated with rearing replacement heifers that have low potential for future milk yield.

Overall, routine genotyping of heifer calves and yearling heifers on commercial farms can be justified. This simulation study provides inferences regarding genetic management decisions on commercial dairy farms, but we could not consider all possible scenarios. If the primary goal is to reduce costs associated with rearing genetically inferior heifers, genotyping calves before 2 mo of age and subsequently selling the poorest animals may be optimal. In contrast, if all heifers will be retained and the primary goal is to use genomic information to make more effective breeding decisions, such as to determine which heifers receive sex-enhanced semen or to identify optimal mating combinations, genotyping heifers at 11 to $12 \mathrm{mo}$ of age may be preferred. The simulation results suggest that routine genotyping of lactating cows that have phenotypic data will be cost effective only in special circumstances, such as when pedigrees are lacking or when testing may result in identification of elite animals that can be marketed as breeding stock or propagated using advanced reproductive technologies. However, strategies based on selective genotyping after presorting by traditional PTA can be somewhat beneficial. A limitation of this simulation study is that it does not consider the potential revenue that commercial dairy producers might realize by using advanced reproductive technologies, such as ET or IVEP, to create additional females that could be marketed as breeding stock or retained to accelerate within-herd genetic progress. Therefore, our results do not reflect full market value of superior breeding stock that may be identified by genomic testing, nor do these results reflect the market or salvage value of inferior animals that were sold from the herd. Traditionally, only pedigree breeders with registered cattle could effectively generate income from the sale of breeding stock or fully realize the benefits of advanced reproduc- tive technologies. However, with the advent of inexpensive SNP arrays for widespread genomic screening, it is likely that many valuable females will be identified in commercial herds that have historically relied solely on income from the sale of milk, and additional marketing benefits could be derived from selective genotyping of lactating cows with superior pedigrees and phenotypes. Last, this simulation did not fully capture the management and labor costs associated with genomic testing, which could be significant on a large commercial dairy farm.

\section{CONCLUSIONS}

The results of this study suggest that routine genomic testing of heifer calves and yearling heifers for the purpose of enhancing the value of future herd replacements can be cost effective, particularly on farms with missing, incomplete, or inaccurate pedigree information. If pedigree or performance data are available, presorting heifers or cows by traditional PA or PTA and genotyping a subset of animals may be a more effective strategy. Results of this study indicate that keeping accurate pedigree and performance data can enhance applications of genomic selection, rather than be replaced by it. Genetic selection is a long-term process, and commercial dairy herds that choose to utilize genomic testing should consider a multi-year planning horizon, so that additional benefits associated with multiple generations of genotyped females can be captured. Developments in genomic selection, such as release of a new version of the Illumina BovineLD chip (which contains 6,909 SNP) and expansion of the Holstein, Jersey, and Brown Swiss reference populations, will enhance the accuracy of genotype imputation and improve predicted breeding values throughout the dairy industry. Developments in herd management that can help producers use information about the genetic predisposition and health history of their heifers and cows more effectively when making breeding, feeding, and culling decisions will also add value to this technology, particularly if such tools are integrated into herd management software programs. Finally, although genomic selection is a new and exciting technology, producers should not neglect existing management practices that are known to enhance farm profitability, such as extensive use of genetically superior AI sires and accurate recording of pedigree and performance data.

\section{ACKNOWLEDGMENTS}

Financial support provided to K. Weigel by the National Association of Animal Breeders (Columbia, 
MO) is gratefully acknowledged, as are helpful comments and suggestions by Nate Zwald of Alta Genetics (Watertown, WI).

\section{REFERENCES}

Casellas, J., and L. Varona. 2011. Short communication: Effect of mutation age on genomic predictions. J. Dairy Sci. 94:4224-4229.

Dassonneville, R., R. F. Brøndum, T. Druet, F. Guillaume, B. Guldbrandtsen, M. S. Lund, V. Ducrocq, and G. Su. 2011. Effect of imputing markers from a low-density chip on the reliability of genomic breeding values in Holstein populations. J. Dairy Sci. 94:3679-3686.

Hansen Axelsson, H., K. Johansson, S. Eriksson, K.-J. Petersson, L. Rydhmer, and J. Philipsson. 2011. Selection of bull dams for production and functional traits in an open nucleus herd. J. Dairy Sci. 94:2592-2600.

Hayes, B. J., P. J. Bowman, A. J. Chamberlain, and M. E. Goddard. 2009. Invited review: Genomic selection in dairy cattle: Progress and challenges. J. Dairy Sci. 92:433-443.

McHugh, N., T. H. E. Meuwissen, A. R. Cromie, and A. K. Sonesson. 2011. Use of female information in dairy cattle genomic breeding programs. J. Dairy Sci. 94:4109-4118.
Van Tassell, C. P., and L. D. Van Vleck. 1991. Estimates of genetic selection differentials and generation intervals for four paths of selection. J. Dairy Sci. 74:1078-1086.

VanRaden, P. M., C. P. Van Tassell, G. R. Wiggans, T. S. Sonstegard, R. D. Schnabel, J. F. Taylor, and F. Schenkel. 2009. Reliability of genomic predictions for North American dairy bulls. J. Dairy Sci. $92: 16-24$

Weigel, K. A., G. de los Campos, A. I. Vazquez, G. J. M. Rosa, D. Gianola, and C. P. Van Tassell. 2010b. Accuracy of direct genomic values derived from imputed single nucleotide polymorphism genotypes in Jersey cattle. J. Dairy Sci. 93:5423-5435.

Weigel, K. A., C. P. Van Tassell, J. R. O'Connell, P. M. VanRaden, and G. R. Wiggans. 2010a. Prediction of unobserved single nucleotide polymorphism genotypes of Jersey cattle using reference panels and population-based imputation algorithms. J. Dairy Sci. 93:2229-2238.

Wensch-Dorendorf, M., T. Yin, H. H. Swalve, and S. König. 2011. Optimal strategies for the use of genomic selection in dairy cattle breeding programs. J. Dairy Sci. 94:4140-4151.

Wiggans, G. R., P. M. VanRaden, and T. A. Cooper. 2011. The genomic evaluation system in the United States: Past, present, future. J. Dairy Sci. 94:3202-3211.

Zhang, Z., and T. Druet. 2010. Marker imputation with low-density markers panels in Dutch Holstein cattle. J. Dairy Sci. 93:54875494. 